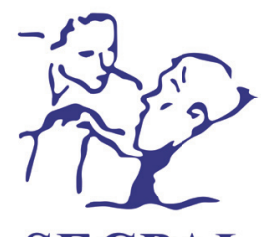

Medicina Paliativa

\title{
Recomendaciones sobre los fármacos empleados en pacientes oncohematológicos afectos de COVID-19. Una aproximación a las interacciones farmacológicas entre el tratamiento sintomático y el tratamiento "anti-COVID-19"
}

\author{
Jennifer Milla-Terrarosa ${ }^{1}$, Kevin Molina Mata², Carmen Muñoz Sánchez ${ }^{3}$ y Marta Val León ${ }^{3}$ \\ ${ }^{1}$ Unidad de Cuidados Paliativos, Instituto Catalán de Oncología. L'Hospitalet de Llobregat, Barcelona, España. ${ }^{2}$ Unidad de \\ Hospitalización de Oncología Médica. Instituto Catalán de Oncología. L'Hospitalet de Llobregat, Barcelona, España. \\ ${ }^{3}$ Servicio de Farmacia. Instituto Catalán de Oncología. L’Hospitalet de Llobregat, Barcelona, España
}

Recibido el 1 de julio de 2020

Aceptado el 19 de septiembre de 2020

\section{PALABRAS CLAVE COVID-19, dolor, delirium, disnea.}

\begin{abstract}
Resumen
La pandemia por SARS-CoV-2 que expuso a nuestros pacientes a una importante carga sintomática hizo de los cuidados paliativos una herramienta necesaria para la atención de estos pacientes. Los fármacos empleados para contrarrestar el virus y los tratamientos para el control de síntomas tienen múltiples interacciones debido tanto al hepatotrofismo del virus como a las vías de metabolismo de los tratamientos.

Con este artículo pretendemos compartir nuestra experiencia y algunas recomendaciones farmacológicas para minimizar potenciales interacciones y efectos secundarios de los tratamientos empleados. Queda mucho por investigar, pero creemos que compartiendo información entre centros podremos brindar el mejor abordaje multidisciplinar que merecen los pacientes.
\end{abstract}

\footnotetext{
*Autor para correspondencia: Jennifer Milla-Terrarosa

Unidad de Cuidados Paliativos, Instituto Catalán de Oncología. Avinguda de la Gran Vía de L’Hospitalet, n. 199-203. 08908, L’Hospitalet de Llobregat, Barcelona, España

Correo electrónico: jennifer.milla@iconcologia.net
} 


\section{KEYWORDS}

COVID-19, pain,

delirium, dyspnoea.

\begin{abstract}
The SARS-CoV-2 pandemic that exposed our patients to a significant symptom burden made palliative care a necessary tool for the care of these patients. The drugs used to counteract the virus and the treatments used for the control of symptoms have multiple interactions due to both the hepatotrophism of the virus and the metabolism pathways of treatments.

With this article we aim to share our experience and some pharmacological recommendations to minimize the potential interactions and side effects of treatments. Much remains to be investigated but we believe that by sharing information between centers we shall be able to provide the best multidisciplinary approach that patients deserve.
\end{abstract}

Milla-Terrarosa J, Molina Mata K, Muñoz Sánchez C, Val León M. Recomendaciones sobre los fármacos empleados en pacientes oncohematológicos afectos de COVID-19. Una aproximación a las interacciones farmacológicas entre el tratamiento sintomático y el tratamiento “anti-COVID-19”. Med Paliat. 2020;27(3):171-180

\section{INTRODUCCIÓN}

La actual pandemia causada por el virus SARS-CoV-2 ha puesto de manifiesto la necesidad de implementar los cuidados paliativos en nuestra práctica diaria. Entre un 15 y un $22 \%$ de los pacientes ingresados por COVID-19 tiene un alto riesgo de morir padeciendo importante sintomatología sistémica por afectación pulmonar grave con disnea severa e insuficiencia respiratoria ${ }^{1,2}$.

Este escenario ha supuesto, en muchos casos, un contexto de cuidados paliativos de emergencia ${ }^{3-5}$. No obstante, el nuevo abordaje terapéutico paliativo requiere de ciertas consideraciones, especialmente si existe concomitancia con determinados tratamientos "anti-COVID-19", como por ejemplo el uso de la hidroxicloroquina. Su administración conjunta con los fármacos utilizados para el control sintomático en cuidados paliativos muestra un alto riesgo de interacciones y efectos secundarios nocivos para los pacientes.

A pesar de las recomendaciones establecidas en 2018 por la Comisión Lancet sobre el Acceso a Cuidados Paliativos y Alivio del Dolor que, en términos generales, pone a la morfina y midazolam como "medicamentos esenciales" en cuidados paliativos $^{6}$, la actual pandemia por SARS-CoV-2 ha supuesto, tanto en España como en otras zonas del mundo, el desabastecimiento de muchos de estos fármacos, generando un gran problema de salud mundial. Así, Pettus y cols. reflejaban el incremento del uso de opioides y benzodiacepinas en pacientes afectos por COVID-19 que requerían de ventilación mecánica. Estas carencias deben verse subsanadas por alternativas seguras, tanto en pacientes tributarios a tratamiento "anti-COVID-19" como en los únicamente candidatos a tratamiento sintomático.

Aunque el mayor esfuerzo terapéutico se ha enfocado en contener la enfermedad y permitir superar la fase más crítica, el cuadro clínico y emocional de los pacientes con peor evolución requiere un abordaje multidimensional e interdisciplinar, mediante la aplicación de medidas efectivas e individualizadas ${ }^{7,8}$.

\section{MATERIALES Y MÉTODOS}

Como centro monográfico oncohematológico que ha tratado pacientes afectos de COVID-19 durante esta pandemia, el presente artículo pretende realizar una serie de recomendaciones sobre el uso de fármacos habituales en cuidados paliativos con asociación del tratamiento "anti-COVID-19". A fecha de hoy, no existen protocolos estandarizados que tengan en cuenta las interacciones farmacológicas y efectos secundarios entre benzodiacepinas, opiáceos, fármacos coadyuvantes, antipalúdicos y antirretrovirales. Para ello, realizamos una exhaustiva revisión bibliográfica considerando los diferentes portales de interacciones farmacológicas y el protocolo de actuación en pacientes COVID-19 aprobado en nuestra institución en marzo de $2020^{\circ}$.

\section{MANEJO DEL CONTROL SINTOMÁTICO EN PACIENTES ONCOHEMATOLÓGICOS AFECTOS DE COVID-19}

Con fecha 14 de marzo de 2020, empezaron a ingresar en nuestro centro pacientes oncohematológicos afectos de COVID-19. En base a las directrices establecidas a nivel de la Agencia Española de Medicamentos y Productos Sanitarios (AEMPS), nuestro hospital consensuó un procedimiento multidisciplinar de actuación para pacientes infectados por SARS-CoV-2 que, entre otros puntos, abordó su manejo terapéutico. Como tratamiento base "anti-COVID-19" se consideraron los siguientes fármacos: hidroxicloroquina, lopinavir/ ritonavir, tocilizumab y remdesivir (Tabla I).

Estos fármacos eran utilizados en monoterapia o de forma combinada en función del estado clínico de los pacientes, según algoritmos de decisión consensuados y siempre previo consentimiento verbal informado del paciente.

En base a los fármacos considerados, se establecieron directrices a aplicar previas a la prescripción y administración de dichos fármacos. Estas incluían la revisión de posi- 
Tabla I. Tratamientos "anti-COVID-19" aprobados en centro monográfico oncohematológico. Marzo de 202010-15

\begin{tabular}{|c|c|c|c|c|}
\hline $\begin{array}{l}\text { Tratamientos } \\
\text { "anti-COVID-19" }\end{array}$ & $\begin{array}{l}\text { Hidroxicloroquina } \\
\text { (HDCQ) }\end{array}$ & $\begin{array}{l}\text { Lopinavir/ritonavir } \\
\text { (LPV/r) }\end{array}$ & $\begin{array}{l}\text { Tocilizumab } \\
\text { (TCZ) }\end{array}$ & $\begin{array}{l}\text { Remdesivir } \\
\text { (RDV) }\end{array}$ \\
\hline $\begin{array}{l}\text { Dosificación } \\
\text { habitual }\end{array}$ & $\begin{array}{l}\text { DC: } 400 \mathrm{mg} / 12 \text { h OR Día } 1 \\
\text { DDM: } 200 \mathrm{mg} / 12 \text { h OR Días 2-5 } \\
\text { Total: } 5 \text { días } \\
\left({ }^{*}\right) \\
\text { Administrar con alimentos o leche }\end{array}$ & $\begin{array}{l}\text { DDM: } 400 / 100 \mathrm{mg} / 12 \mathrm{~h} \mathrm{OR} \\
\text { Total: } 5 \text { - } 10 \text { días } \\
\left({ }^{* *}\right) \\
\text { Administrar con o sin } \\
\text { alimentos }\end{array}$ & $\begin{array}{l}\text { Peso } \geq 75 \mathrm{~kg}: \text { DU } 600 \mathrm{mg} \text { EV } \\
\text {-Peso < } 75 \mathrm{~kg}: \text { DU } 400 \mathrm{mg} \text { EV } \\
\left.{ }^{* * *}\right) \\
\text { Diluir en } 50-100 \mathrm{~mL} \text { SSF. } \\
\text { Administrar en infusión } \\
\text { intermitente de } 1 \text { hora }\end{array}$ & $\begin{array}{l}\text { DC: } 200 \mathrm{mg} \text { EV Día } 1 \\
\text { DDM: } 100 \mathrm{mg} \text { EVI Días } 2-10 \text { Total: } 10 \\
\text { días } \\
\text { Reconstituir vial } 100 \mathrm{mg} \text { con } 20 \mathrm{ml} \text { de } \\
\text { API } \\
\text { Diluir en } 100-250 \mathrm{ml} \text { SSF } \\
\text { Administrar en infusión de } 30 \text { minutos }\end{array}$ \\
\hline $\begin{array}{l}\text { Dosificación } \\
\text { insuficiencia renal }\end{array}$ & $\begin{array}{l}\mathrm{ClCr}<10 \mathrm{ml} / \mathrm{min}: \text { administrar } 100 \% \mathrm{DC} . \\
\text { Continuar con } 50 \% \text { DDM }\end{array}$ & $\begin{array}{l}\text { No requiere ajuste de dosis } \\
\text { Precaución con solución oral } \\
\text { (riesgo acumulación de } \\
\text { propilenglicol) }\end{array}$ & No requiere ajuste de dosis & $\begin{array}{l}\mathrm{ClCr}>30 \mathrm{ml} / \mathrm{min}: \text { administrar } 100 \% \text { de } \\
\mathrm{DC} \text { y DDM } \\
\mathrm{ClCr}<30 \mathrm{~mL} / \mathrm{min}: \text { no administrar }\end{array}$ \\
\hline $\begin{array}{l}\text { Dosificación } \\
\text { insuficiencia } \\
\text { hepática }\end{array}$ & $\begin{array}{l}\text { Precaución. Puede requerir ajuste de dosis. } \\
\text { No directrices concretas }\end{array}$ & $\begin{array}{l}\text { Precaución. Puede requerir } \\
\text { ajuste de dosis. No } \\
\text { directrices concretas. Evitar } \\
\text { en insuficiencia hepática } \\
\text { grave }\end{array}$ & $\begin{array}{l}\text { Precaución. No directrices } \\
\text { concretas. Evitar si ALT y/o AST } \\
>5 \times \text { LSN }\end{array}$ & $\begin{array}{l}\text { Precaución. } \\
\text { ALT } \geq 5 \text { LSN: no administrar } \\
\text { Evitar si ALT alterada y síntomas de } \\
\text { inflamación hepática o aumento de Bil } \\
\text { conjugada, FA o índice internacional } \\
\text { normalizado (IIN) }\end{array}$ \\
\hline $\begin{array}{l}\text { Principales efectos } \\
\text { secundarios }\end{array}$ & $\begin{array}{l}\text { Cardiacos: prolongación QT, TdP, insuficiencia } \\
\text { cardiaca, cardiomiopatía, etc. } \\
\text { Gastrointestinales: náuseas, vómitos, } \\
\text { diarreas, etc. } \\
\text { Cefalea, vértigos, pérdida audición, } \\
\text { fotofobia, reducción agudeza visual, etc. } \\
\text { Hipoglucemia, con o sin tratamiento } \\
\text { antidiabético concomitante } \\
\text { Otros: musculoesqueléticos y hematológicos } \\
\text { en tratamientos prolongados }\end{array}$ & $\begin{array}{l}\text { Gastrointestinales: náuseas, } \\
\text { vómitos, diarreas, etc. } \\
\text { Cardiacos: prolongación QT, } \\
\text { TdP, etc. } \\
\text { Hipertrigliceridemia e } \\
\text { hipercolesterolemia } \\
\text { Pancreatitis }\end{array}$ & $\begin{array}{l}\text { Infecciones de tracto } \\
\text { respiratorio superior, } \\
\text { nasofaringitis, cefalea, } \\
\text { hipertensión arterial y } \\
\text { elevación de transaminasas } \\
\text { hepáticas (ALT y/o AST), etc. } \\
\text { Otros: infecciones graves, } \\
\text { complicaciones de la } \\
\text { diverticulitis y reacciones de } \\
\text { hipersensibilidad }\end{array}$ & $\begin{array}{l}\text { Hepáticos: elevación de transaminasas } \\
\text { Gastrointestinales: náuseas, vómitos, } \\
\text { etc. } \\
\text { Cefalea, hipotensión, erupción } \\
\text { cutánea, etc. }\end{array}$ \\
\hline Contraindicaciones & $\begin{array}{l}\text { Hipersensibilidad a: principio activo, } \\
\text { derivados de } 4 \text {-aminoquinolinas o alguno de } \\
\text { los excipientes incluidos } \\
\text { Déficit de glucosa-6-fosfato deshidrogenasa } \\
\text { Porfiria } \\
\text { Miastenia gravis }\end{array}$ & $\begin{array}{l}\text { Hipersensibilidad a: } \\
\text { principios activos o alguno } \\
\text { de los excipientes incluidos }\end{array}$ & $\begin{array}{l}\text { Neutrófilos }<500 \text { células } / \mathrm{mm}^{3} \\
\text { Plaquetas }<50.000 \text { células } / \\
\mathrm{mm} 3 \\
\text { Sepsis documentada por } \\
\text { patógeno diferente a SARS- } \\
\text { CoV-2 }\end{array}$ & $\begin{array}{l}\text { Hipersensibilidad a principio activo } \\
\text { No administrar de forma concomitante } \\
\text { con cloroquina o hidroxicloroquina por } \\
\text { riesgo de disminución de actividad de } \\
\text { remdesivir }\end{array}$ \\
\hline \multirow[t]{2}{*}{ Recomendaciones } & \multicolumn{2}{|c|}{$\begin{array}{l}\text { Controlar intervalo QT P Realizar ECG antes de iniciar, durante y después de } \\
\text { finalizar el tratamiento. QTc normal: mujeres < } 470 \mathrm{~ms} \text {; hombres < } 440 \mathrm{~ms} \text {. } \\
\text { Evitar QTC }>500 \mathrm{~ms} \text {. Adecuar tratamiento según procedimiento aprobado }\end{array}$} & $\begin{array}{l}\text { Diverticulitis complicada o } \\
\text { perforación intestinal } \\
\text { Infección cutánea en curso }\end{array}$ & $\begin{array}{l}\text { Controlar función hepática renal antes } \\
\text { y durante el tratamiento }\end{array}$ \\
\hline & \multicolumn{4}{|c|}{$\begin{array}{l}\text { Revisar interacciones farmacológicas con medicación crónica o de nueva prescripción P Adecuar tratamiento según información obtenida y } \\
\text { procedimiento aprobado. }\end{array}$} \\
\hline
\end{tabular}


bles interacciones farmacológicas tanto con la medicación crónica de base del paciente, como con los tratamientos de nueva prescripción, incluyendo fármacos para el control sintomático, si procedía.

En la Tabla II quedan reflejadas las interacciones farmacológicas entre los medicamentos utilizados para el control sintomático y los fármacos "anti-COVID-19" prescritos. Las recomendaciones tienen en cuenta nuestra experiencia clínica y lo contrastado con diferentes portales de interacciones medicamentosas.

En la Tabla III se muestran propuestas de tratamientos "alternativos".

Cabe mencionar que, debido al conocimiento progresivo que hemos ido obteniendo sobre la infección por SARS-CoV-2 y su manejo, algunas de las recomendaciones mencionadas pueden quedar obsoletas.

\section{Dolor}

El control sintomático del dolor en cuidados paliativos según recomendaciones del "Manual del control de Síntomas en Pacientes con cáncer" de l'Institut Català d' Oncologia, incluye fármacos como: analgésicos no opioides (paracetamol, antinflamatorios no esteroideos [AINE]), opioides y coanalgésicos.

Respecto a los analgésicos no opioides, al inicio de la pandemia se creía que el consumo de AINE podía empeorar la evolución de los pacientes infectados por el virus SARSCoV-2, según la hipótesis planteada por Fang y cols. Postulaba que los AINE podían aumentar los niveles del receptor de la enzima convertidora de angiotensina 2 (ECA2), puerta principal de entrada del virus a las células epiteliales de nuestro organismo ${ }^{16}$. Así, paracetamol llegó a considerarse la alternativa segura al ibuprofeno en caso de automedicación. Posteriormente, tanto la Organización Mundial de la Salud (OMS) como la AEMPS desmintieron esta hipótesis por falta de datos objetivos, regularizándose la utilización de los AINE en la población afectada por COVID-19 y considerando para su manejo aspectos farmacocinéticos, efectos secundarios e interacciones potenciales con tratamientos "anti-COVID-19"17.

Centrándonos en el uso de opioides, morfina y fentanilo en sus vías de administración habituales, son los fármacos de elección iniciándose a dosis bajas y posterior titulación hasta alcanzar la dosis terapéutica diana. Por ejemplo, iniciar con dosis de morfina un $50 \%$ menos de lo habitual $(2,5 \mathrm{mg}$ cada 4 horas) sería una conducta segura tal como lo reflejamos en la Tabla III. En pacientes COVID-19 debemos considerar dos puntos fundamentales en su manejo: a) el hepatotrofismo del virus ${ }^{18}$ y su frecuente asociación a la alteración en el perfil hepático del paciente infectado, condicionando la utilización de opioides de mayor metabolización hepática (morfina); b) las posibles interacciones farmacológicas con los tratamientos "anti-COVID-19" administrados (alteraciones del intervalo QTc [metadona], concentraciones plasmáticas mayores a las esperadas con dosis estándares de opioides [morfina, fentanilo, etc.]), llegando a limitar, en algunos casos, la selección de opioides comunes y generando la necesidad de buscar alternativas terapéuticas no habituales, pero más seguras en este ámbito, como tapentadol o remifentanilo, en base a las interacciones potenciales descritas.
En cuanto a los coanalgésicos indicados en el dolor neuropático, se incluyen antidepresivos (amitriptilina, duloxetina o venlafaxina) y antiepilépticos (carbamazepina, gabapentina, pregabalina o clonazepam). Algunos de estos fármacos se asocian a alteraciones del intervalo QTc (antidepresivos), así como a una farmacocinética con intensa metabolización hepática (carbamazepina), pudiendo generar importantes efectos secundarios por la concomitancia con tratamientos "anti-COVID-19" y/o por la presencia de disfunción hepática en el paciente. Por ello, en la población de pacientes COVID-19 se recomienda básicamente gabapentina o pregabalina, a dosis habituales, como las opciones más seguras en dolor neuropático.

\section{Disnea}

En nuestra experiencia, y en la de otros equipos hospitalarios responsables del cuidado de pacientes COVID-19, hemos observado que esta población suele expresar un malestar general y/o astenia intensa que, junto a la hipoperfusión periférica e hipoxemia (objetivada mediante pulsioximetría), nos deben orientar a interpretarlo y tratarlo como disnea.

El manejo sintomático de la disnea puede abordarse con opioides como primera línea de tratamiento, pero la oxigenoterapia suplementaria, los corticoesteroides y las benzodiazepinas también pueden coadyuvar ${ }^{19}$.

En los cuidados paliativos, ante disnea asociada a proceso oncohematológico, se recomienda iniciar de forma precoz los opiáceos con titulación hasta alcanzar un control del síntoma. Entre los opiáceos, la morfina es el fármaco de elección. En la concomitancia COVID-19 y paliativos, los mórficos siguen siendo el fármaco ideal, pero se valorará el inicio del tratamiento con dosis menores a las habituales.

Respecto al rol de los corticoesteroides en la infección por SARS-CoV-2, la información publicada durante la pandemia ha sido muy controvertida. Se postula que el uso de estos fármacos durante los primeros días de la infección (cuando la carga viral es mayor) podría enlentecer la eliminación del virus, agravando así la infección ${ }^{20,21}$. Esta afirmación se basa en la experiencia que hemos obtenido con otros virus de características similares (SARS-CoV, MERS-CoV e Influenza). Por lo tanto, deberíamos intentar evitar la corticoterapia en la primoinfección, pudiendo plantear su inicio en etapas más avanzadas cuando cobra mayor protagonismo la respuesta inflamatoria. En este punto, la corticoterapia podría aportar un beneficio adicional a nivel respiratorio por su potente efecto antinflamatorio ${ }^{22,23}$. Su utilización en población COVID-19 puede verse potenciada en concomitancia con determinados tratamientos "anti-COVID-19", debiendo valorar su beneficio clínico respecto al riesgo asociado y posible relevancia clínica.

Las benzodiazepinas pueden estar indicadas en la disnea como tratamiento complementario a los opiáceos considerando su acción ansiolítica. Son de gran utilidad en las crisis de disnea intensas con componente de ansiedad asociada, siendo midazolam el fármaco de elección en población convencional. No obstante, las benzodiazepinas son fármacos que presentan una gran metabolización hepática, y como sucede con otros fármacos ya descritos, su concomitancia con determinados tratamientos “anti-COVID” puede provo- 
Recomendaciones sobre los fármacos empleados en pacientes oncohematológicos afectos de COVID-19.

Tabla II. Interacciones farmacológicas entre fármacos usados en el control sintomático y fármacos específicos antiCOVID-19. Procedimiento aprobado en centro oncohematológico ${ }^{10-15}$.

\begin{tabular}{|c|c|c|c|c|}
\hline $\begin{array}{l}\text { Analgésicos No } \\
\text { Opioides }\end{array}$ & $\begin{array}{c}\text { Lopinavir/ritonavir } \\
(\mathrm{LPV} / \mathrm{r})\end{array}$ & Hidroxicloroquina (HDCQ) & Tocilizumab (TCZ) & $\begin{array}{l}\text { Remdesivir } \\
\text { (RDV) }\end{array}$ \\
\hline $\begin{array}{l}\text { Ácido } \\
\text { acetilsalicílico }\end{array}$ & Interacción poco probable & Interacción poco probable & $\begin{array}{l}\text { Interacción poco } \\
\text { probable }\end{array}$ & $\begin{array}{l}\text { Interacción poco } \\
\text { probable }\end{array}$ \\
\hline Diclofenaco & Interacción poco probable & Interacción poco probable & $\begin{array}{l}\text { Interacción poco } \\
\text { probable }\end{array}$ & $\begin{array}{l}\text { Interacción poco } \\
\text { probable }\end{array}$ \\
\hline Ibuprofeno & Interacción poco probable & Interacción poco probable & $\begin{array}{l}\text { Interacción poco } \\
\text { probable }\end{array}$ & $\begin{array}{l}\text { Interacción poco } \\
\text { probable }\end{array}$ \\
\hline Indometacina & Interacción poco probable & Interacción poco probable & $\begin{array}{l}\text { Interacción poco } \\
\text { probable }\end{array}$ & $\begin{array}{l}\text { Interacción poco } \\
\text { probable }\end{array}$ \\
\hline Ketorolaco & Interacción poco probable & Interacción poco probable & $\begin{array}{l}\text { Interacción poco } \\
\text { probable }\end{array}$ & $\begin{array}{l}\text { Interacción poco } \\
\text { probable }\end{array}$ \\
\hline Metamizol & Riesgo de $\downarrow$ Cp de LPV/r & $\begin{array}{l}\text { Riesgo de toxicidad } \\
\text { hematológica }\end{array}$ & $\begin{array}{l}\text { Riesgo de toxicidad } \\
\text { hematológica }\end{array}$ & $\begin{array}{l}\text { Interacción poco } \\
\text { probable }\end{array}$ \\
\hline Naproxeno & Interacción poco probable & Interacción poco probable & $\begin{array}{l}\text { Interacción poco } \\
\text { probable }\end{array}$ & $\begin{array}{l}\text { Interacción poco } \\
\text { probable }\end{array}$ \\
\hline Paracetamol & Interacción poco probable & Interacción poco probable & $\begin{array}{l}\text { Interacción poco } \\
\text { probable }\end{array}$ & $\begin{array}{l}\text { Interacción poco } \\
\text { probable }\end{array}$ \\
\hline $\begin{array}{l}\text { Analgésicos } \\
\text { opioides menores }\end{array}$ & Lopinavir/ritonavir (LPV/r) & Hidroxicloroquina (HDCQ) & Tocilizumab (TCZ) & \\
\hline Codeína & $\begin{array}{l}\text { Riesgo de } \downarrow \text { Cp codeína. } \\
\text { Pueden requerirse } \uparrow \text { dosis } \\
\text { codeína }\end{array}$ & $\begin{array}{l}\text { Riesgo de } \downarrow \text { Cp codeína. Pueden } \\
\text { requerirse } \uparrow \text { dosis codeína }\end{array}$ & $\begin{array}{l}\text { Interacción poco } \\
\text { probable }\end{array}$ & $\begin{array}{l}\text { Interacción poco } \\
\text { probable }\end{array}$ \\
\hline Tramadol & $\begin{array}{l}\text { Riesgo de } \uparrow \text { Cp tramadaol. } \\
\text { Pueden requerirse } \downarrow \text { dosis } \\
\text { tramadol } \\
\text {-Monitorizar signos de } \\
\text { toxicidad }\end{array}$ & $\begin{array}{l}\text { Riesgo prolongación QT + } \\
\text { hipoglucemia. Pueden } \\
\text { requerirse } \downarrow \text { dosis tramadol } \\
\text {-Monitorizar glucosa + ECG + } \\
\text { signos de toxicidad }\end{array}$ & $\begin{array}{l}\text { Interacción poco } \\
\text { probable }\end{array}$ & $\begin{array}{l}\text { Interacción poco } \\
\text { probable }\end{array}$ \\
\hline $\begin{array}{l}\text { Analgésicos } \\
\text { opioides mayores }\end{array}$ & Lopinavir/ritonavir (LPV/r) & Hidroxicloroquina (HDCQ) & Tocilizumab (TCZ) & \\
\hline Buprenorfina & $\begin{array}{l}\text { Riesgo prolongación QT. } \\
\text { Riesgo } \uparrow \text { Cp buprenorfina. } \\
\text { Pueden requerirse } \downarrow \text { dosis } \\
\text { buprenorfina } \\
\text {-Monitorizar ECG + signos } \\
\text { de toxicidad }\end{array}$ & $\begin{array}{l}\text { Riesgo Prolongación QT } \\
\text {-Monitorizar ECG }\end{array}$ & $\begin{array}{l}\text { Interacción poco } \\
\text { probable }\end{array}$ & $\begin{array}{l}\text { Interacción poco } \\
\text { probable }\end{array}$ \\
\hline Fentanilo & $\begin{array}{l}\text { Evitar. Riesgo de } \uparrow \uparrow \mathrm{Cp} \\
\text { fentanilo. Si es } \\
\text { imprescindible, utilizar } \downarrow \downarrow \\
\text { dosis fentanilo } \\
\text {-Monitorizar signos de } \\
\text { toxicidad }\end{array}$ & Interacción poco probable & $\begin{array}{l}\text { Interacción poco } \\
\text { probable }\end{array}$ & $\begin{array}{l}\text { Interacción poco } \\
\text { probable }\end{array}$ \\
\hline Hidromorfona & $\begin{array}{l}\text { Riesgo de } \uparrow \mathrm{Cp} \\
\text { hidromorfona. Pueden } \\
\text { requerirse } \downarrow \text { dosis } \\
\text { hidromorfona. } \\
\text {-Monitorizar signos de } \\
\text { toxicidad. }\end{array}$ & Interacción poco probable & $\begin{array}{l}\text { Interacción poco } \\
\text { probable }\end{array}$ & $\begin{array}{l}\text { Interacción poco } \\
\text { probable }\end{array}$ \\
\hline Morfina & $\begin{array}{l}\text { Riesgo } \uparrow \text { Cp metabolito } \\
\text { activo. Pueden requerirse } \\
\downarrow \text { dosis morfina. } \\
\text {-Monitorizar signos de } \\
\text { toxicidad. }\end{array}$ & Interacción poco probable & $\begin{array}{l}\text { Interacción poco } \\
\text { probable }\end{array}$ & $\begin{array}{l}\text { Interacción poco } \\
\text { probable }\end{array}$ \\
\hline
\end{tabular}


Tabla II (Cont.). Interacciones farmacológicas entre fármacos usados en el control sintomático y fármacos específicos anti-COVID-19. Procedimiento aprobado en centro oncohematológico ${ }^{10-15}$.

\begin{tabular}{|c|c|c|c|c|}
\hline $\begin{array}{c}\text { Analgésicos No } \\
\text { Opioides }\end{array}$ & Lopinavir/ritonavir (LPV/r) & Hidroxicloroquina (HDCQ) & Tocilizumab (TCZ) & $\begin{array}{l}\text { Remdesivir } \\
\text { (RDV) }\end{array}$ \\
\hline Metadona & $\begin{array}{l}\text { Evitar. Riesgo prolongación } \\
\text { QT. Riesgo } \downarrow \downarrow \text { Cp metadona. } \\
\text { Si es imprescindible, } \\
\text { individualizar dosis } \\
\text { metadona. Combinación } \\
\text { estudiada } \\
\text {-Monitorizar ECG + síndrome } \\
\text { de abstinencia }\end{array}$ & $\begin{array}{l}\text { Evitar. Riesgo Prolongación QT. } \\
\text { Si es imprescindible, utilizar } \downarrow \downarrow \\
\text { dosis metadona } \\
\text {-Monitorizar ECG }\end{array}$ & $\begin{array}{l}\text { Interacción poco } \\
\text { probable }\end{array}$ & $\begin{array}{l}\text { Interacción poco } \\
\text { probable }\end{array}$ \\
\hline Oxicodona & $\begin{array}{l}\text { Evitar. Riesgo } \uparrow \uparrow \mathrm{Cp} \\
\text { oxicodona. Pueden requerirse } \\
\downarrow \downarrow \text { dosis oxicodona. Si es } \\
\text { imprescindible, utilizar } \downarrow \downarrow \\
\text { dosis oxicodona } \\
\text {-Monitorizar signos de } \\
\text { toxicidad }\end{array}$ & $\begin{array}{l}\text { Riesgo } \uparrow \text { Cp oxicodona. Pueden } \\
\text { requerirse } \downarrow \text { dosis oxicodona } \\
\text {-Monitorizar signos de toxicidad }\end{array}$ & $\begin{array}{l}\text { Interacción poco } \\
\text { probable }\end{array}$ & $\begin{array}{l}\text { Interacción poco } \\
\text { probable }\end{array}$ \\
\hline Tapentadol & Interacción poco probable & Interacción poco probable & $\begin{array}{l}\text { Interacción poco } \\
\text { probable }\end{array}$ & $\begin{array}{l}\text { Interacción poco } \\
\text { probable }\end{array}$ \\
\hline Remifentanil & Interacción poco probable & Interacción poco probable & $\begin{array}{l}\text { Interacción poco } \\
\text { probable }\end{array}$ & $\begin{array}{l}\text { Interacción poco } \\
\text { probable }\end{array}$ \\
\hline
\end{tabular}

Cp: concentraciones plasmáticas. AUC: área bajo la curva.

car graves efectos secundarios. Precisamente, midazolam es la benzodiazepina con mayor repercusión en su utilización combinada con determinados tratamientos "anti-COVID-19". Presenta un extenso metabolismo hepático, mediado casi exclusivamente por la isoenzima CYP3A4 del citocromo $\mathrm{P} 450$, pudiendo interaccionar con algunos tratamientos "anti-COVID-19" que actúan como potentes inhibidores de dicha isoenzima, acentuando sus efectos clínicos en caso de concomitancia, pudiendo llegar a generar sedación extrema y/o parada respiratoria. Aunque estas interacciones son mucho más evidentes con la administración de midazolam vía oral, por su efecto de primer paso hepático, el resto de vías de administración también se encuentran afectadas.

Por tanto, en población COVID-19, será muy importante conocer el grado de afectación de la concomitancia entre las benzodiazepinas más comunes y el tratamiento "antiCOVID-19" utilizado, así como las medidas o alternativas a contemplar, siendo lorazepam y lormetazepam las benzodiazepinas más seguras.

La oxigenoterapia está indicada en el paciente con disnea e hipoxemia. En ausencia de hipoxemia su uso es controvertido, pero puede ser de utilidad valorar su empleo si el paciente percibe mejoría sintomática. Si nuestro paciente requiere de un mayor soporte ventilatorio, el uso de la ventilación mecánica no invasiva (VMNI) podría estar indicada y es factible administrarla empleando cánulas nasales o máscaras faciales. Antes de indicarlo hay que evaluar integralmente al paciente considerando su pronóstico vital, beneficio clínico esperado, deseo y valores del paciente. Esta terapia sintomática puede dar tiempo a que el tratamiento "antiCOVID-19" actúe y/o a la toma de decisiones. Su uso debe ser individualizado y necesita de personal con experiencia en su manejo, ya que supone un mayor riesgo de contagio por la mayor difusión del virus en la sala de tratamiento ${ }^{24-26}$.

\section{Tos}

La tos irritativa, junto con la disnea, es otro síntoma común durante la infección por SARS-CoV-2. Si es muy incapacitante, tratamientos a corto plazo con fármacos habitualmente utilizados en población convencional (codeína y morfina a dosis bajas) pueden mejorar los síntomas ${ }^{27}$. No obstante, debemos saber que la codeína se metaboliza a nivel hepático por la enzima CYP2D6, originando morfina como metabolito activo. Determinados tratamientos "antiCOVID-19" pueden inhibir dicha enzima, pudiendo reducir su efecto antitusígeno y requiriendo de dosis mayores a las habituales.

\section{Depresión y ansiedad}

Existe un alto riesgo de que la temporalidad brusca e incapacitante del cuadro de SARS-CoV-2 limite las posibilidades de iniciar antidepresivos. Dadas las interacciones farmacológicas, podemos vernos obligados a "modificar" pautas antidepresivas por la seguridad de los pacientes. Según las recomendaciones estipuladas, desvenlafaxina, venlafaxina y duloxetina son de los antidepresivos con menores interacciones farmacológicas. Incluso podría no descontinuarse reduciendo la dosis terapéutica habitual un $50 \% 28$. 
Recomendaciones sobre los fármacos empleados en pacientes oncohematológicos afectos de COVID-19.

Tabla III. Propuesta de tratamientos "alternativos" para control sintomático en población COVID-19 y tratamiento "anti-COVID-19". Basado en procedimiento aprobado en centro monográfico oncohematológico ${ }^{10-15}$.

\begin{tabular}{|c|c|c|c|}
\hline Síntoma & $\begin{array}{c}\text { Tratamiento "Alternativo } \\
\text { Seguro" }\end{array}$ & $\begin{array}{c}\text { "Propuesta" Dosificación Inicial + Ajuste } \\
\text { Posterior }\end{array}$ & Consideraciones Especiales \\
\hline \multirow{4}{*}{$\begin{array}{l}\text { Dolor } \\
\text { nociceptivo }\end{array}$} & $\begin{array}{l}\text { Paracetamol +/- AINE } \\
\text { (FF de liberación normal) }\end{array}$ & $\begin{array}{l}\text { Dosis y pautas habituales OR o EV } \\
\text { Según fármaco } \rightarrow \text { Considerar DMD } \\
\text { habituales }\end{array}$ & $\begin{array}{l}\text { Según fármaco } \rightarrow \text { Ajustes } \\
\text { habituales en } I R, \text { IH o } \geq 65 \text { años } \\
\text { Evitar metamizol }\end{array}$ \\
\hline & $\begin{array}{l}\text { Tapentadol } \\
\text { (FF de liberación prolongada) }\end{array}$ & $\begin{array}{l}\text { PI: } 25 \text { mg/ } 12 \text { h OR. Posteriormente, } \uparrow 50 \\
\text { mg/12 h OR cada } 3 \text { días } \\
\text { DMD: } 500-600 \text { mg }\end{array}$ & $\begin{array}{l}\text { Precaución en } \mathrm{PI} \text { en } \geq 65 \text { años e } \\
\text { IH moderada } \\
\text { Evitar en IR e IH graves }\end{array}$ \\
\hline & $\begin{array}{l}\text { Remifentanilo } \\
\text { (FF de liberación normal) }\end{array}$ & $\begin{array}{l}\text { PI inducción 0,5-1 mg/kg/min EV } \\
\text { Posteriormente, PM: 0,05-0,1 mg/kg/min } \\
\text { EV (ICEV) } \\
\text { DMD: } 0,2 \mu \mathrm{g} / \mathrm{kg} / \mathrm{min} \\
\text { Tramitar como indicación fuera de ficha } \\
\text { técnica }\end{array}$ & $\begin{array}{l}\mathrm{PI} \text { en } \geq 65 \text { años: } 50 \% \mathrm{PI} \text { adultos. } \\
\text { Posteriormente, ?según } \\
\text { respuesta y tolerancia } \\
\text { No requiere ajuste en IR. } \\
\text { Precaución en IH grave }\end{array}$ \\
\hline & $\begin{array}{l}\text { Morfina } \\
\text { (FF de liberación normal + } \\
\text { prolongada) }\end{array}$ & $\begin{array}{l}\text { Utilizar } 50 \% \text { dosis habituales OR o SC o } \\
\text { EV. Considerar EQ V Adm } \\
\text { PI: } 2,5-5 \mathrm{mg} / 4 \text { h OR }\left(^{*}\right)+\text { DE si dolor }=1 / 6 \\
\text { DTD } \\
\text { Posteriormente, } \uparrow \text { DTD según DE } \\
\text { requeridas }\left({ }^{\wedge}\right)\end{array}$ & $\begin{array}{l}\text { PI en } \geq 65 \text { años, fragilidad o IR: } \\
2,5 \mathrm{mg} / 8-6 \mathrm{~h} \text { OR } \\
\text { Evitar en IH grave }\end{array}$ \\
\hline \multirow[t]{2}{*}{$\begin{array}{l}\text { Dolor } \\
\text { neuropático }\end{array}$} & $\begin{array}{l}\text { Gabapentina } \\
\text { (FF de liberación normal) }\end{array}$ & $\begin{array}{l}\text { PI: } 300 \text { mg UVD día } 1,300 \text { mg DVD día } 2 \text { y } \\
300 \text { mg TVD día } 3 \text { OR } \\
\text { Posteriormente, } \uparrow \text { DTD en } 300 \text { mg/día } \\
\text { cada } 2-3 \text { días, repartida en } 3 \text { tomas, según } \\
\text { respuesta y tolerancia, hasta alcanzar DEF } \\
\text { DMD: } 1.800 \text { mg en } 1 \text { semana; } 2.400 \text { mg en } \\
2 \text { semanas; } 3.600 \text { mg en un total de } 3 \\
\text { semanas }\end{array}$ & $\begin{array}{l}\mathrm{PI} \text { en } \geq 65 \text { años o fragilidad } \\
\text { puede ser menor y más lenta } \\
\text { Considerar } \mathrm{DMD} \text { y ajustes } \\
\text { posología en } \mathrm{CICr}<80 \mathrm{~mL} / \mathrm{min} \\
\pm \mathrm{HD} \\
\text { No requiere ajuste en } \mathrm{IH}\end{array}$ \\
\hline & $\begin{array}{l}\text { Pregabalina } \\
\text { (FF de liberación normal) }\end{array}$ & $\begin{array}{l}\text { PI: } 25-75 \text { mg/día OR } \\
\text { Posteriormente, } \uparrow 75 \mathrm{mg} / \text { día hasta } 300 \\
\mathrm{mg} / \text { día en 3-7 días, repartida en } 2-3 \\
\text { tomas } \\
\text { DMD: } 600 \mathrm{mg} \text { en un total de } 2 \text { semanas, } \\
\text { repartida en 2-3 tomas }\end{array}$ & $\begin{array}{l}\text { Precaución en } \geq 65 \text { años } \\
\text { Considerar } \mathrm{DMD} \text { y ajustes } \\
\text { posología en } \mathrm{ClCr}<60 \mathrm{~mL} / \mathrm{min} \\
\pm \mathrm{HD} \\
\text { No requiere ajuste en } \mathrm{IH}\end{array}$ \\
\hline \multirow{3}{*}{ Disnea } & $\begin{array}{l}\text { Morfina } \\
\text { (FF de liberación normal) }\end{array}$ & $\begin{array}{l}\text { Utilizar } 50 \% \text { dosis habituales OR o SC o } \\
\text { EV. Considerar EQ V Adm } \\
\mathrm{PI}: 1,25-2,5 \mathrm{mg} / 4 \mathrm{~h} \text { OR } \\
\text { Si uso previo de opioides, } \uparrow \text { DTD en un } \\
25-50 \% \\
\text { Si crisis de disnea, administrar DE } \\
\text { equivalente SC }\end{array}$ & $\begin{array}{l}\text { PI en } \geq 65 \text { años, fragilidad o IR: } \\
1,25 \mathrm{mg} / 8-6 \mathrm{~h} \text { OR } \\
\text { Evitar en IH grave }\end{array}$ \\
\hline & $\begin{array}{l}\text { Lorazepam } \\
\text { (FF de liberación normal) }\end{array}$ & $\begin{array}{l}\mathrm{PI}: 0,5-2 \mathrm{mg} / \text { día SL } \\
\mathrm{DMD}: 4 \mathrm{mg}\end{array}$ & $\begin{array}{l}\text { PI en } \geq 65 \text { años o fragilidad: } \\
0,25 \mathrm{mg} / \text { día SL, con DMD: } 2 \mathrm{mg} \\
\text { No requiere ajuste en IR } \\
\text { Precaución en IH grave }\end{array}$ \\
\hline & $\begin{array}{l}\text { Dexametasona } \\
\text { (FF de liberación normal) }\end{array}$ & $\begin{array}{l}\mathrm{PI}: 6-8 \mathrm{mg} / \text { día OR } \\
\text { Posteriormente, } \uparrow \text { hasta } 10-12 \mathrm{mg} / \text { día si } \\
\text { no respuesta. Suspender en } 7-10 \text { días }\end{array}$ & $\begin{array}{l}\text { Precaución en } \geq 65 \text { años o IH } \\
\text { grave } \\
\text { No requiere ajuste en IR }\end{array}$ \\
\hline \multirow[t]{2}{*}{ Tos } & $\begin{array}{l}\text { Codeína } \\
\text { (FF de liberación normal) }\end{array}$ & $\begin{array}{l}\text { PI: } 15-30 \mathrm{mg} / 6 \mathrm{~h} \text { OR } \\
\text { DMD: } 240 \mathrm{mg}\end{array}$ & $\begin{array}{l}\text { Precaución en } \geq 65 \text { años, } \\
\text { fragilidad o IH } \\
\text { Considerar DMD y ajustes } \\
\text { posología en } \mathrm{ClCr}<60 \mathrm{ml} / \mathrm{min}\end{array}$ \\
\hline & $\begin{array}{l}\text { Morfina } \\
\text { (FF de liberación normal) }\end{array}$ & $\begin{array}{l}\text { Utilizar } 50 \text { \% dosis habituales OR o SC o } \\
\text { EV. Considerar EQ V Adm } \\
\text { PI: } 1,25-2,5 \mathrm{mg} / 4-6 \text { h OR }\end{array}$ & $\begin{array}{l}\text { PI en } \geq 65 \text { años, fragilidad o IR: } \\
1,25 \mathrm{mg} / 8-6 \mathrm{~h} \text { OR } \\
\text { Evitar en } \mathrm{IH} \text { grave }\end{array}$ \\
\hline
\end{tabular}


Tabla III (Cont.). Propuesta de tratamientos “alternativos" para control sintomático en población COVID-19 y tratamiento “anti-COVID-19”. Basado en procedimiento aprobado en centro monográfico oncohematológico10-15.

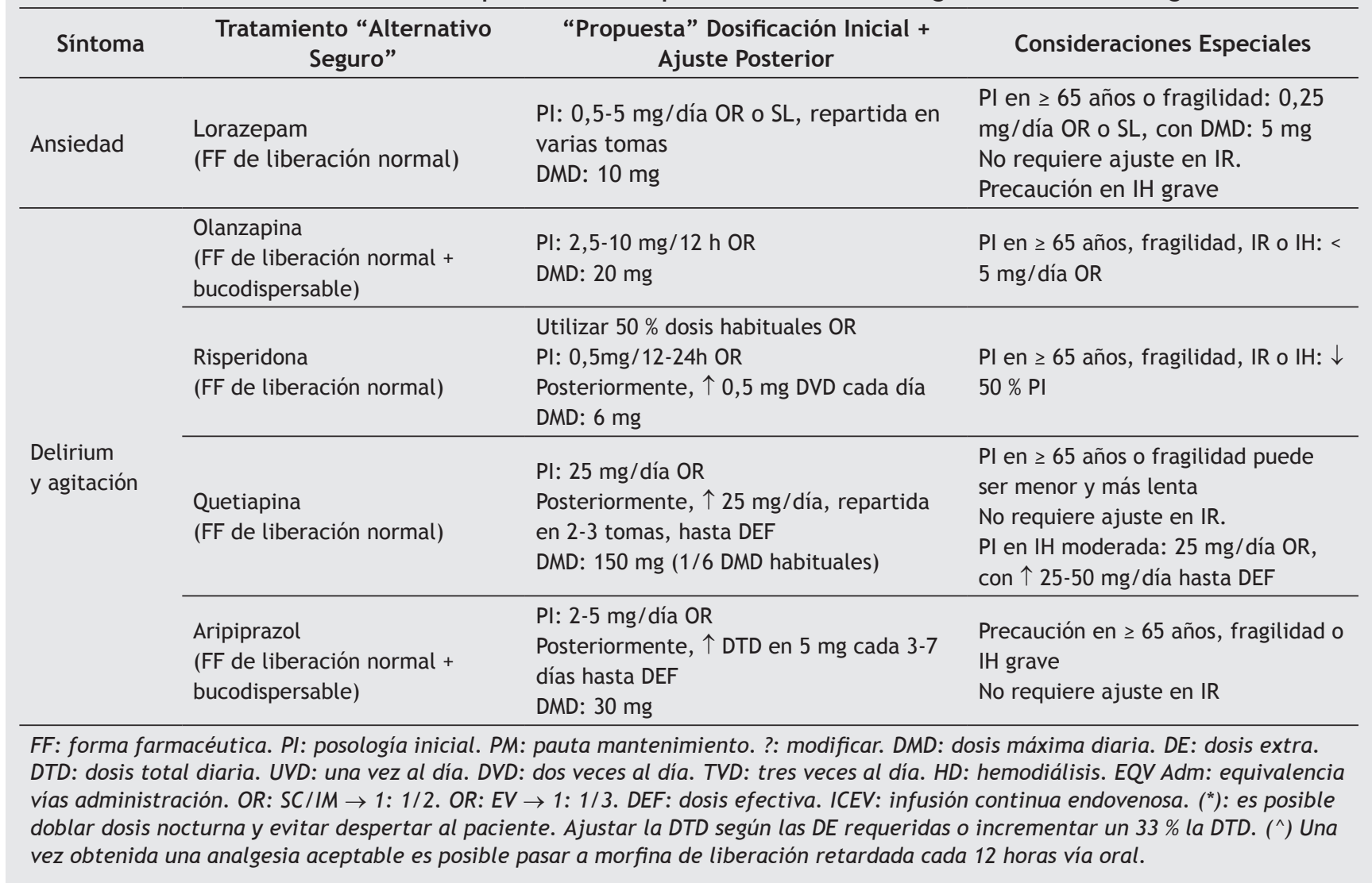

Si la manifestación clínica predominante es la ansiedad, se recomienda como primera opción ansiolítica el lorazepam, siempre que el paciente pueda asegurar la vía oral.

\section{Delirium}

El delirium o síndrome confusional agudo (SCA) es el trastorno cognitivo más frecuente en cuidados paliativos en cáncer, llegando a presentar una incidencia de un 86 en pacientes con cáncer avanzado ${ }^{29}$.

El paciente con infección por SARS-CoV-2 presenta un alto riesgo de desarrollar un SCA durante su estancia hospitalaria. Factores como el aislamiento, la restricción de visitas (imposibilitando la estimulación sensorial, estrategias de comunicación o de mitigación del estrés emocional), la hipoxia generada por la insuficiencia respiratoria, el fallo orgánico, así como el propio neurotropismo de los Coronaviridae generan, de manera sinérgica, un ambiente idóneo para el desarrollo del delirium hiperactivo con inquietud y ansiedad. Está reportado que hasta un $15 \%$ de los pacientes hospitalizados con COVID-19 presenta alguna manifestación de delirium ${ }^{30,31} \mathrm{e}$ incluso que su presentación puede traducirse en mal pronóstico a corto plazo, como sucede en el caso de pacientes críticos ingresados en la Unidad de Cuidados Intensivos (UCI) ${ }^{32}$.

En población convencional, como parte del abordaje terapéutico de este síndrome, siempre se deben intentar identi- ficar posibles causas reversibles que lo originen e instaurar medidas no farmacológicas de reorientación. En caso de no conseguir su reconducción, el tratamiento farmacológico a iniciar irá orientado a controlar el síntoma más incapacitante. En caso de delirium hiperactivo, los neurolépticos típicos (haloperidol, levomepromazina, clorpromazina) y atípicos (quetiapina risperidona u olanzapina, administrados vía oral) se consideran los fármacos de elección. En casos de delirium con agitación refractaria, midazolam endovenoso es el fármaco de elección.

En población COVID-19 con determinado tratamiento "anti-COVID-19", la mayoría de estos medicamentos pueden presentar importantes limitaciones. La utilización de haloperidol en concomitancia con determinados fármacos "antiCOVID-19" estaría contraindicada por alto riesgo de Torsades de pointes y muerte súbita, especialmente en su administración vía intravenosa. Para quetiapina también existe un alto riesgo arritmogénico, aunque en algunas guías se recomienda su utilización a dosis mucho menores a las habituales (1/6 dosis habitual). La utilización de risperidona también estaría controvertida en concomitancia con determinadas combinaciones de tratamientos "anti-COVID-19”, sugiriéndose evitar su utilización o bien utilizarla a dosis inferiores a las habituales, si es estrictamente necesario. Olanzapina sería la alternativa terapéutica más segura, siendo una buena opción en agitación aguda, considerando especialmente su administración mediante comprimidos bucodispersables. 
Según la bibliografía consultada, otro fármaco que podría utilizarse en delirium con agitación en concomitancia con tratamientos “anti-COVID-19”, sería aripiprazol28, neuroléptico atípico de administración oral que puede utilizarse a dosis estándares o reducidas al $50 \%$.

Otros fármacos como diazepam o levomepromazina también podrían utilizarse en delirium con agitación en población COVID-19, dependiendo del tratamiento concomitante "anti-COVID-19" y considerando la reducción de dosis habituales en algunos casos. En este contexto, también existe alguna experiencia reportada, como la de Lovell y cols. ${ }^{33}$, con la administración segura de midazolam a dosis no superiores a $10 \mathrm{mg}$ en infusión continua intravenosa.

\section{CONCLUSIONES}

Los pacientes afectos de COVID-19 experimentan una gran carga sintomática. Entre un 15 y un $22 \%$ de ellos presenta una enfermedad de curso clínico agresivo llegando a ser letal por la importante afectación pulmonar.

Los cuidados paliativos son una parte esencial del manejo para responder a esta pandemia, independientemente del nivel de intervención terapéutica que requiera cada paciente.

Un $20 \%$ de los pacientes infectados por SARS-CoV-2 debido a la gravedad clínica requieren ingreso en $\mathrm{UCI}$. De estos, el 50 \% fallece, por lo que deberían beneficiarse de una atención paliativa integral precoz.

Los tratamientos contra el COVID-19 orientados a frenar la enfermedad y la medicación estándar indicada para controlar los síntomas más incapacitantes deben monitorizarse estrechamente debido a sus posibles interacciones farmacológicas. Pruebas complementarias como: función renal, hepática, parámetros inflamatorios, ionograma y/o electrocardiogramas (control del intervalo QTc) nos facilitarán tener un control óptimo.

Disponemos de poca evidencia relacionada con los cuidados paliativos en pacientes afectos de COVID-19. Por ello, es necesario seguir investigando en los síntomas desarrollados por esta población y su manejo, así como proporcionar una buena formación a los profesionales sanitarios para poder definir un plan terapéutico óptimo, considerando sus necesidades, preservando sus deseos y su calidad de vida.

\section{BIBLIOGRAFÍA}

1. Zhou F, Yu T, Du R, Fan G, Liu Y, Liu Z, et al. Clinical course and risk factors for mortality of adult inpatients with COVID-19 in Wuhan, China: a retrospective cohort study. Lancet. 2020;395:1054-62.

2. Guan W-j, Liang W-h, Zhao Y, Liang HR, Chen ZS, Yi YM, et al. Comorbidity and its impact on 1,590 patients with COVID-19 in China: A Nationwide Analysis. Eur Respir J. 2020;55:2000547.

3. Rubio O, Estella A, Cabre L, Saralegui-Reta I, Martin MC, Zapata $\mathrm{L}$, et al. Recomendaciones éticas para la toma de decisiones difíciles en las unidades de cuidados intensivos ante la situación excepcional de crisis por la pandemia por covid-19: revisión rápida y consenso de expertos. Med Intensiva. 2020. DOI: $10.1016 /$ j.medin.2020.04.006.
4. Verity R, Okell LC, Dorigatti I, Winskill P, Whittaker C, Imai N, et al. Estimates of the severity of coronavirus disease 2019: a model-based analysis. Lancet Infect Dis. 2020;20:669-77.

5. Tanja F-S, Nancy P, Keller N, Gamondi C. Conservative management of Covid-19 patients - emergency palliative care in action. J Pain Symptom Manage. 2020;60(1):e27-e30.

6. Pettus K, Cleary JF, de Lima L, Ahmed E, Radbruch L. Availability of International Controlled Essential Medicines in the COVID-19 Pandemic. J Pain Symptom Manage. 2020;60(2):e48e51.

7. SECPAL. Orientaciones sobre el control sintomático de enfermos graves afectados por la enfermedad covid 19 y que requieran atención paliativa o se encuentren próximos al final de la vida [Internet]. Sociedad Española de Cuidados Paliativos; 2020. Disponible en: https: //www.secpal.com/biblioteca_documentos-covid19-1.

8. Domenico BG, Claudia G, Monika O, Ralf J. CoviD-19: Decision making and palliative care. Swiss Med Wkly. 2020;150:2-3.

9. Institut Catala d'Oncología. Procediment d'actuació davant de la sospita i la infecció per SARS-CoV-2 en pacients a l'Institut Català d'Oncologia de L'Hospitalet [Internet]. Generalitat de Catalunya; Març de 2020. Disponible en: https: / / scientiasalut. gencat.cat/bitstream/handle/11351/4684/procediment_actuacio_inicial_davant_sospita_infeccio_coronavirus_sars_ cov2_2020.pdf?sequence=4\&isAllowed =y.

10. Portal Drug Interaction Checker [Internet]. Medscape; 2020. Disponible en: https://reference.medscape.com/drug-interactionchecker

11. Portal Drug Interactions Checker [Internet]. Drugs.com; 2020. Disponible en: https://www.drugs.com/

12. Recomendaciones del grupo de interacciones medicamentosas COVID-19 Drug Interactions [Internet]. Universidad de Liverpool; 2020. Disponible en: https://www.covid19-druginteractions.org/

13. Portal de interacciones [Internet] Lexicomp $® ; 2020$. Disponible en: https://online.lexi.com/lco/action/home

14. Base de datos [Internet]. UpToDate $® ;$ 2020. Disponible en: https://www.uptodate.com/contents/search

15. Fichas técnicas de medicamentos de la AEMPS [Internet]. Ministerio de Sanidad; 2020. Disponible en: https://cima.aemps. es/cima/publico/home.html

16. Fang $L$, Karakiulakis $G$, Roth $M$. Are patients with hypertension and diabetes mellitus at increased risk for COVID-19 infection? Lancet Respir Med. 2020;8:e21.

17. Agencia Española de Medicamentos y Productos Sanitarios. La AEMPS informa que ningún dato indica que el ibuprofeno agrave las infecciones por COVID-19 [Internet]. Ministerio de Sanidad; 15 de marzo de 2020. Disponible en: https: / /www.aemps.gob. es/informa/notasinformativas/medicamentosusohumano-3/2020-medicamentosusohumano-3/la-aemps-informa-queningun-dato-indica-que-el-ibuprofeno-agrave-las-infeccionespor-covid-19/

18. Su T-H, Kao J-H. The clinical manifestations and management of COVID-19-related liver injury. J Formos Med Assoc. 2020;119:1016-8.

19. Strieder M, Pecherstorfer M, Kreye G. Symptomatic treatment of dyspnea in advanced cancer patients: A narrative review of the current literature. Wien Med Wochensch. 2018;168:333-43.

20. Russell CD, Millar JE, Baillie JK. Clinical evidence does not support corticosteroid treatment for 2019-nCoV lung injury. Lancet. 2020;395:473-5.

21. Lianhan Shang, Jianping Zhao, Yi Hu, Ronghui Du, Bin Cao. On the use of corticosteroids for 2019-nCoV pneumonia. Lancet. 2020;395:683-4.

22. Zhou W, Liu Y, Tian D, Wang C, Wang S, Cheng J, et al. Potential benefits of precise corticosteroids therapy for severe 2019-nCoV pneumonia. Signal Transduct Target Ther. 2020;5:17-9. 
23. Zhou YH, Qin YY, Lu YQ, Sun F, Yang S, Harypursat V, et al. Effectiveness of glucocorticoid therapy in patients with severe novel coronavirus pneumonia: protocol of a randomized controlled trial. Chin Med J (Engl). 2020;133:1080-6.

24. Winck JC, Ambrosino N. COVID-19 pandemic and non invasive respiratory management: every Goliath needs a David. An evidence based evaluation of problems. Pulmonology. 2020;26:21320.

25. Cinesi Gómez C, Peñuelas Rodríguez O, Luján Torné ML, Egea Santaolalla C, Masa Jiménez JF, García Fernández J, et al. Clinical consensus recommendations regarding non-invasive respiratory support in the adult patient with acute respiratory failure secondary to SARS-CoV-2 infection. Rev Esp Anestesiol Reanim (English Edition). 2020;67:261-70.

26. Arulkumaran N, Brealey D, Howell D, Singer M. Use of non-invasive ventilation for patients with COVID-19: a cause for concern? Lancet Respir Med. 2020;8:e45.

27. National Institute for Health and Care Excellence (NICE) in collaboration with NHS England and NHS Improvement. Managing COVID-19 symptoms (including at the end of life) in the community: summary of NICE guidelines. BMJ. 2020;369:m1461.
28. Manejo farmacológico de trastornos psicóticos en personas mayores con tratamiento d la infección por COVID19: interacciones y recomendaciones terapéuticas [Internet]. Sociedad Española de Medicina Interna; 2020. Disponible en: https: / /www. fesemi. org/sites/default/files/documentos/19.pdf.

29. Julia-Torras J, Serrano Bermúdez G. Manual de Control de Síntomas en Pacientes con Cáncer. $4 .^{\text {a }}$ ed. Madrid: Arán Ediciones S.L.; 2019.

30. Mao L, Jin H, Wang M, Hu Y, Chen S, He Q, et al. Neurologic manifestations of hospitalized patients with Coronavirus Disease 2019 in Wuhan, China. JAMA Neurol. 2020;77:683-90.

31. Kotfis K, Williams Roberson S, Wilson JE, Dabrowski W, Pun BT, Ely EW. COVID-19: ICU delirium management during SARS-CoV-2 pandemic. Crit Care. 2020;24:176.

32. ICNARC. Report on COVID-19 in critical care. Intensive Care Natl Audit Res Cent [Internet]. 2020;(April):1-24.

33. Lovell N, Maddocks M, Etkind SN, Taylor K, Carey I, Vora V, et al. Characteristics, symptom management and outcomes of 101 patients with COVID-19 referred for hospital palliative care. J Pain Symptom Manage. 2020;60:e77-e81. 